\title{
Increased circulating GrMyeloid-derived suppressor cells correlated with tumor burden and survival in locally advanced cervical cancer patient
}

\author{
Yun Liang ${ }^{1}$,Bingjian Lü1,2,Peng Zhao ${ }^{3}$, and Weiguo L $\ddot{u ̈}^{3}$ \\ 1. Department of Surgical Pathology, the Women's Hospital, School of Medicine, Zhejiang University. Zhejiang Province 310006, China \\ 2. Center for Uterine Cancer Diagnosis \& Therapy of Zhejiang Province, Zhejiang Province 310006, China \\ 3. Department of Gynecology, the Women's Hospital, School of Medicine, Zhejiang University. Zhejiang Province 310006, China \\ $\square$ Corresponding author: Dr. Weiguo Lü, Professor, Department of Gynecology, the Women's Hospital, School of Medicine, Zhejiang University. 2 Xueshi \\ Road, Hangzhou 310006, Zhejiang, China, Email: lbwg@zju.edu.cn, Tel: +86-0571-89991702 \\ (C) Ivyspring International Publisher. This is an open access article distributed under the terms of the Creative Commons Attribution (CC BY-NC) license \\ (https://creativecommons.org/licenses/by-nc/4.0/). See http://ivyspring.com/terms for full terms and conditions.
}

Received: 2018.09.01; Accepted: 2019.01.10; Published: 2019.01.30

\begin{abstract}
Background: Myeloid-derived suppressor cells (MDSCs) may lead to immune evasion and disease progression in some cancers, but their roles remain to be investigated in cervical cancer. This study aimed to characterize the specific subsets of MDSCs that might be potential indicators for disease progression in cervical cancer patients.

Methods: Different subsets of MDSCs were characterized using flow cytometry in blood samples from healthy subjects and cervical cancer patients. T-cell proliferation assay was performed in vitro. Tumor infiltrating CD8+ and FOXP3+ cells were also evaluated by immunostaining on the tumor tissues from patients with early and locally advanced cervical cancer. We further analyzed the relevance of circulating MDSCs to clinicopathological characteristics, patient survival as well as tumor infiltrating lymphocytes.

Results: The frequency of circulating GrMDSCs increased correlated with FIGO stage while the percentage of MoMDSCs only elevated in patients with advanced cervical cancers. For patients with early and locally advanced cervical cancer, the frequency of circulating GrMDSCs but not MoMDSCs correlated with clinicopathologic parameters including metastatic lymph nodes, deep stromal invasion and tumor recurrence. The levels of circulating GrMDSCs also negatively correlated with the densities of CD8+ cells in tumor tissues. In vitro assay showed that Gr-MDSCs suppressed the proliferation of autologous $\mathrm{T}$ cells.

Conclusion: Our study demonstrates that the increased frequency of circulating GrMDSCs is associated with tumor burden and recurrence in early and locally advanced cervical cancer patients, that the GrMDSCs may be potential biomarkers for disease progression cervical cancers.
\end{abstract}

Key words: cervical squamous cell carcinoma; myeloid-derived suppressor cells; recurrence; tumor infiltrating lymphocytes

\section{Background}

Cancer development is largely determined by the intrinsic cellular changes and immunologic factors in the tumor macro- and microenvironment. Suppression of tumor immune surveillance is one of the main mechanisms that facilitate tumor cells to escape from destruction by the immune system. Ample evidence supports a key role for
Myeloid-derived suppressor cells (MDSCs) in immune suppression in tumor development growth and progression [1]. MDSCs represent a heterogenic population of mixed immature bone marrow-derived myeloid cells. These cells are characterized by some phenotypic markers, depending on the differentiation context by factors secreted from cancer cells. In 
mouse, granulocytic MDSCs (GrMDSCs) have a CD11b+Ly6G+Ly6Clow phenotype whereas monocytic MDSCs (MoMDSCs) are CD11b+Ly6GLy6Chigh [2]. Human MDSCs lack the Gr1 like antigens and remain to be characterized biologically and phenotypically. MDSCs from cancer patients express the common myeloid marker CD33 and CD11b, but lack mature myeloid or lymphoid markers, such as HLA-DR. As in murine, human MDSCs have been allocated into three predominant populations [3,4]. MoMDSCs which morphologically resemble monocytes express CD14 but usually not CD15. GrMDSCs share morphological characteristics with granulocytes and express CD15 but lack CD14. Immature MDSCs represent the least differentiation population and lack expression of CD14 or CD15.

A few studies have investigated the population of MDSCs in cervical cancers. In vitro, a population of MDSCs defined as: Lin-CD33+HLADRlow was induced from healthy donor peripheral bloods by co-culture with HeLa cells [5]. Recently, one research had described significantly elevated levels of CD11b+ CD33+ HLA-DR- MDSCs cells, in the peripheral bloods of 14 advanced and recurrent cervical cancer patients [6]. However, due to the limited number of patients, the MDSC subpopulations and their clinical relevance remain unclear to date. In this study, we have undertaken a comprehensive flow cytometry-based study to investigate the phenotypes of circulating MDSCs in patients with cervical cancer. We hope to identify potential subtypes which could be served as a potential biomarker for cancer progression.

\section{Methods}

\section{Study population}

The Institutional Review Board approved the study. Forty cervical cancer patients (FIGO stage Ia-IIa) with complete clinical data were recruited in Women's Hospital, School of Medicine, Zhejiang University, China from January 2015 to June 2015.The patients had a histological confirmed squamous cervical carcinoma and did not receive any treatment. Blood samples from 11 advanced cervical cancer patients (FIGO stage IIb-IIIb) were also obtained at their first visits. Fifteen age-matched healthy donors were used as controls. Written consents were obtained from all patients before blood sampling. Table 1 shows the clinical features of the patients in this study. For 24 patients with FIGO stage Ib2 and IIa2, $1 / 3$ received platinum-based new adjuvant chemotherapy (NACT), followed by radical hysterectomy, while other early and local advanced cervical cancer patients underwent primary surgery
( $\mathrm{n}=32$ ). Clinical response to chemotherapy was assessed according to WHO criteria. Whole bloods and cervical biopsies before surgery or NACT were used as baseline in cervical cancer patients.

Table 1. The clinical characteristics of patients with cervical cancers

\begin{tabular}{llll}
\hline & $\begin{array}{l}\text { Early stage } \\
(\mathrm{n}=16)\end{array}$ & $\begin{array}{l}\text { Locally advanced } \\
\text { stage }(\mathrm{n}=24)\end{array}$ & $\begin{array}{l}\text { Advanced stage } \\
(\mathrm{n}=11)\end{array}$ \\
\hline FIGO stage & stageIa-Ib1 & (stage Ib2-IIa) & (stage IIb-III) \\
Age $(<40:>40)$ & $4: 12$ & $5: 19$ & $3: 8$ \\
LVSI (yes : no) & $5: 11$ & $10: 14$ & - \\
$\begin{array}{l}\text { Histological grade } \\
(\text { I-II:III) }\end{array}$ & $15: 1$ & $21: 3$ & - \\
$\begin{array}{l}\text { Stromal invasion } \\
(<2 / 3:>2 / 3)\end{array}$ & $11: 5$ & $15: 9$ & - \\
LN metastasis (yes : no) & $3: 13$ & $5: 19$ & - \\
\hline
\end{tabular}

\section{Whole Blood Staining and MDSC immunophenotyping}

Peripheral bloods $(2-3 \mathrm{ml})$ were collected from patients and were processed within 6 hours. Two hundred microliters $(\mu \mathrm{L})$ of whole blood taken for MDSC analysis were divided into $100 \mu \mathrm{L}$ for nonstained tube and $100 \mu \mathrm{L}$ for stained tube with different MDSC markers. To determine the frequency and phenotype of MDSCs in freshly whole blood, a combination of six surface markers including lineage-specific mixture (CD3/CD19/CD56/CD14/ CD20/CD16)-APC, HLADR-APC-Cy7, CD33-PECY7, CD11b-PerCP, CD15-FITC, CD14-PE was used. Samples were vortexed well and incubated for 25 minutes at $4{ }^{\circ} \mathrm{C}$ then with lysis buffer (BD FACS lysing solution) for 15 minutes at room temperature to lyse the red blood cells. Flow cytometric analysis was performed on BD FACS Canto II flow cytometer. Data were analyzed using FlowJo software (TreeStar, Inc, Ashland, OR).

\section{Cell sorting}

Peripheral blood mononuclear cells (PBMCs) were isolated from $15 \mathrm{~mL}$ of freshly obtained blood from two cervical cancer patients and one health donor by Ficoll density gradient centrifugation as previously described. Cell viability analysis was performed on the single-cell suspension of blood PBMCs using trypan blue staining method. Effector cells (CD33+CD14- MDSC cells) were isolated from the PBMCs of patients using MACS microbeads and columns (Miltenyi Biotec, Germany). Briefly, CD14cells were separated by CD14 MicroBeads. CD14-CD33+ cells were further sorted by CD33 MicroBeads from purified CD14- cells. The CD3+ T cells were prepared from PBMCs in the same patients using Human Pan $\mathrm{T}$ cells Isolation Kit (Miltenyi Biotec). The purity of the cells after sorting was $>90 \%$. 


\section{T-cell proliferation assay}

Carboxyfluorescein succinimidy ester (CFSE)based proliferation assays were performed according to manufacturer's instructions (Invitrogen, Carlsbad, CA). T cells (100,000/well) were incubated with CFSE and stimulated with anti-human CD3 and CD28 (both $1 \mathrm{ug} / \mathrm{mL}$ ). No MDSCs or 50,000 (1:2) autologous CD33+CD14- MDSC cells were added to the stimulated $\mathrm{T}$ cells. After stimulation for 3 days, at $37^{\circ} \mathrm{C}, 5 \% \mathrm{CO} 2$, proliferation assay was analyzed by flow cytometry using mAbs: FITC- conjugated CD8 (BD Bioscience). $\mathrm{T}$ cells without anti-CD3 and anti-CD28 antibody stimulation were as negative control.

\section{Immunohistochemistry}

Cervical biopsy specimens were fixed in 10\% neutral buffered formalin, embedded in paraffin and sectioned. For immunohistochemical analysis, $4 \mu \mathrm{m}$ sections were deparaffinized and immunostained using antibodies specific for CD8 (Abcam) or FOXP3 (Abcam). Tumor infiltrating lymphocyte (TIL) density was evaluated microscopically. The number of lymphoid infiltrates was counted manually including 5 tumor beds and 5 peripheries in the biopsy materials. The average counts of lymphoid infiltrates within tumor bed and within lymphoid infiltrates surrounding tumor were recorded separately.

\section{Follow-up and Statistical analysis}

Cases were followed up by letters or telephone. Follow-up time ranged from 19 to 26 months (median 24 months).Disease-free survival (DFS) was defined as the time from treatment to first appearance of recurrence or death. Assumed that the number of MDSCs or TILs did not follow a normal distribution, comparisons between different groups were made using the Mann-Whiney $U$ test. Correlation analyses were performed using Spearman's rank correlation test. The frequencies in NAC groups were analyzed using unpaired $t$ test. Statistical analysis was performed on SPSS version 13.0 for Windows (SPSS Inc., Chicago, IL, USA). For all tests, two-sided $\mathrm{p}<0.05$ was considered to be statistically significant.

\section{Results}

\section{The frequency of MDSCs increased in blood of patients}

We aimed to explore all three subsets of circulating MDSCs using six-color flow cytometric analysis: HLA-DR- Lin- CD11b+ CD33+ CD14CD15-immature MDSCs (a), HLA-DR- Lin- CD11b+ CD33+ CD15+ CD14-GrMDSCs (b) and CD14+ HLADR-/low MoMDSCs (c) in whole blood from cervical cancer patients and age-matched healthy controls. Cervical cancers were divided into early stage (stageIa-Ib1, stage Ib2-IIa with a maximum diameter of $\leq 4 \mathrm{~cm}$ ), locally advanced stage (stage Ib2-IIa with a maximum diameter of $>4 \mathrm{~cm}$ ) and advanced stage (stage IIb-III), respectively. The frequency of circulating MDSCs was calculated as a percentage of total cells. Representative flow cytometric data of a healthy donor and one patient with stage Ib2 are shown in Fig. 1.

A significantly higher level of circulating GrMDSCs in cervical cancer patients were observed compared with controls (mean $3.987 \%$ vs. $0.8669 \%$, $p=0.0001$ ). When patients were further divided by clinical cancer stage, a direct correlation between clinical cancer stage and percentage of circulating GrMDSCs was noted (Spearman's r $=0.794$, $p<0.001)$. There was a statistical difference in GrMDSCs levels between advanced and locally advanced $(p=0.0002)$, early and locally advanced cancer patients $(p=0.0002)$, but was not significant between early stage patients and controls $(p=0.7073)$ (Fig. 2A).

The frequency of circulating MoMDSCs was significantly higher in cancer patients than that in controls (mean $1.324 \%$ vs., $0.3635 \%, p=0.0021$ ). The level of MoMDSCs only increased in patients with advanced cervical cancers $(p<0.0001)$, but not between local advanced and early patients $(p=0.5528)$, early patients and controls ( $p=0.1605)$ (Fig. 2B).

For immature MDSCs, analysis showed that these HLA-DR- Lin- CD11b+ CD33+ cells in peripheral blood samples were almost CD15+. Therefore in peripheral blood of cervical cancer patients, the phenotype of immature MDSCs was virtually non-existent.

\section{Frequency of GrMDSCs but not MoMDSCs correlated with clinicopathologic parameters and survival for early and locally advanced cervical cancers}

The association between the frequency of MDSCs and clinical parameters was shown in Table 2. GrMDSCs levels significantly increased in patients with metastatic lymph nodes (mean $2.503 \%$ vs. $5.006 \%, p=0.0056$ ) and deep stromal invasion (mean $2.234 \%$ vs. $4.435 \%, p=0.0012$ ). No significant correlation was found between the levels of MoMDSCs and clinicopathological parameters in cervical cancer patients. Eight of 24 patients with locally advanced cervical cancers received NAC and 2 achieved pathological optimal response (pOR) after NAC. The frequency of GrMDSCs before NAC tended to be lower in patients with pOR than those without pOR (mean $2.152 \%$ vs. $4.030 \%, p=0.213$ ), but the 
difference did not reach statistical significance due to the small sample size.

Forty patients with early and locally advanced cervical cancers had a median follow-up of 2 years. Five women $(12.5 \%)$ recurred. The frequency of
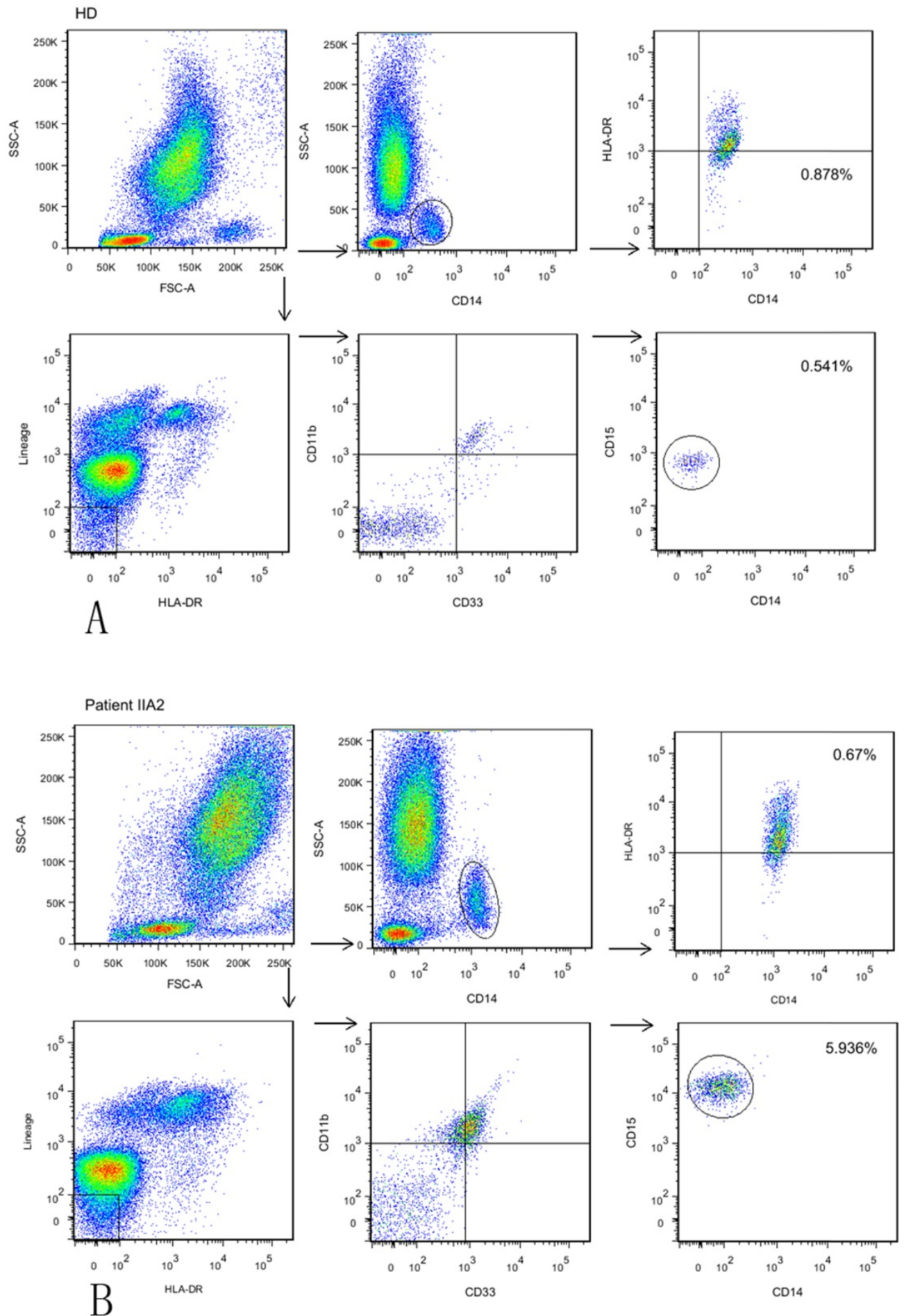

Fig 1. Identification and characterization of different subtype of MDSCs in bloods from GC patients. (A)The frequency of GrMDSCs is $0.541 \%$, and the frequency of MoMDSCs is $0.878 \%$ in a healthy donor. Gating strategy for MDSCs: For immature MDSCs and GrMDSCs, acquired cells were first gated on the non-expression of Lin and HLADR. Within this population, the fraction of cells expressing both CD33 and CD1 lb was determined. The expression of CD15 was further explored in this fraction. For MoMDSCs, we first gated on SSC versus CD14 to isolate the monocytes cell populations, then HLADR versus CD14 was plotted for MoMDSCs. (B) The frequency of GrMDSCs is 5.936\%, and the frequency of MoMDSCs is $0.670 \%$ in a stage II a2 patient 
Table 2. The correlation between MDSCs level and clinicopathologic parameters.

\begin{tabular}{llllll}
\hline & \multicolumn{3}{c}{ GrMDSCs } & MoMDSCs \\
\hline FIGO stage & Ia-Ib1 & mean & $\mathrm{p}$ & mean & $\mathrm{p}$ \\
& Ib2-IIa & $3.472 \%$ & 0.0002 & $0.8131 \%$ & 0.5528 \\
LN metastasis & yes & $2.503 \%$ & 0.0056 & $0.692 \%$ & 0.0863 \\
& no & $5.006 \%$ & & $1.237 \%$ & \\
Histological & III & $3.017 \%$ & 0.9461 & $0.907 \%$ & 0.6684 \\
grade & I-II & $3.889 \%$ & & $0.660 \%$ & \\
Stromal invasion & $>2 / 3$ & $4.435 \%$ & 0.0012 & $1.068 \%$ & 0.2048 \\
\multirow{2}{*}{ Age (year) } & $>2 / 3$ & $2.234 \%$ & & $0.836 \%$ & \\
& $>40$ & $2.943 \%$ & 0.4964 & $0.787 \%$ & 0.1363 \\
& $<40$ & $3.214 \%$ & & $1.213 \%$ & \\
\hline
\end{tabular}

Table 3. The correlations between circulating MDSCs and tumor infiltrating CD8+ and FOXP3+ cells.

\begin{tabular}{lllllllll}
\hline & \multicolumn{2}{l}{$\begin{array}{l}\text { Intraltumoral } \\
\text { CD8+ }\end{array}$} & \multicolumn{2}{l}{$\begin{array}{l}\text { Peritumoral } \\
\text { CD8+ }\end{array}$} & \multicolumn{2}{l}{$\begin{array}{l}\text { Intraltumoral } \\
\text { FOXP3+ }\end{array}$} & \multicolumn{2}{l}{$\begin{array}{l}\text { Peritumoral } \\
\text { FOXP3+ }\end{array}$} \\
\hline r & $\mathrm{p}$ & $\mathrm{r}$ & $\mathrm{p}$ & $\mathrm{r}$ & $\mathrm{p}$ & $\mathrm{r}$ & $\mathrm{p}$ \\
GrMDSCs & -0.401 & 0.010 & -0.355 & 0.025 & 0.189 & 0.244 & 0.132 & 0.416 \\
MoMDSCs & -0.195 & 0.228 & -0.256 & 0.111 & 0.111 & 0.497 & -0.056 & 0.730 \\
\hline
\end{tabular}

\section{Circulating GrMDSCs correlated with the densities of CD8+ cells infiltration in tumor microenvironment}

Both CD8+ cells and Foxp3+ cells displayed a disseminated manner in cervical cancer (Supplementary Fig. S1). Table 3 showed the densities of CD8+ and FOXP3+ cells in the biopsies from 40 early and locally advanced cervical cancer patients. TILs were divided into high levels and low levels according to the median numbers. The circulating GrMDSCs showed weak negative linear relationship to both intratumoral CD8+ cells $(\mathrm{r}=-0.401, p=0.010)$ and peritumoral CD8+ cells $(\mathrm{r}=-0.355, \quad p=0.025)$ densities. However, the levels of circulating MoMDSCs were not associated with the densities of TILs. The densities of Foxp3+ cells had no correlation with both circulating GrMDSCs and MoMDSCs.

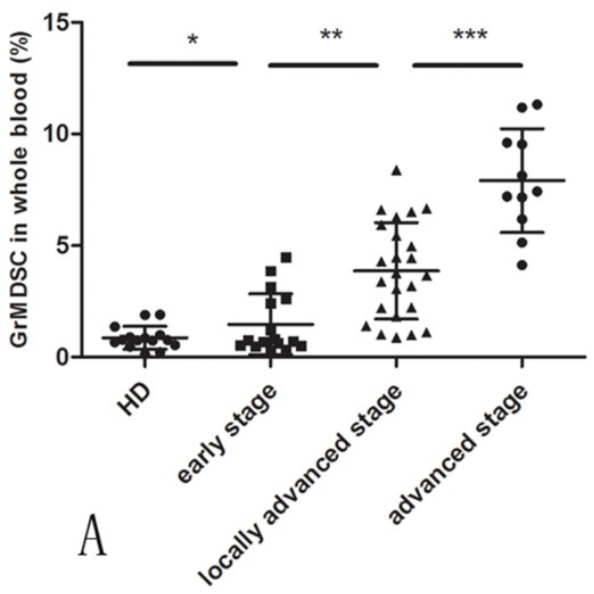

GrMDSCs inhibit T cell proliferation in vitro

As only GrMDSCs levels were correlated with clinical-pathologic parameters and tumor infiltrating lymphocytes, we further investigated the immunosuppressive function of GrMDSCs in vitro. GrMDSCs were sorted from one healthy donor and two patients with stage IIa2 cancer (Fig 3). The sorted CD33+CD14- GrMDSCs from the two patients substantially suppressed the proliferation of autologous $\mathrm{T}$ cells with the proliferation index reducing to $39.9 \%$ and $58.9 \%$, respectively. However, CD33+CD14-GrMDSCs cells from healthy control did not have the inhibitory effects with a $\mathrm{T}$ cell proliferation index of $91.6 \%$.

\section{Discussion}

Cervical cancer is the fourth leading cause of cancer-related deaths among women worldwide and occurs as the result of an uncontrolled persistent high-risk HPV infection due to the failure of effective $\mathrm{T}$ cell response $[7,8]$. Immunosuppressive cells are critical in the loss of immune effects. Among these cells, MDSCs can interfere with $\mathrm{T}$ cell-mediated responses and play an important role immune evasion in cancers.

In this study, we investigated the percentage, phenotype and immunosuppressive function of circulating MDSCs in a cohort of patients with cervical cancers. We found that the level of MDSCs was significantly increased in the blood of patients with advanced cervical cancer. Kawano et al. [6] reported a statistically significant higher frequency of circulating MDSCs in the blood of advanced and recurrent patients with cervical cancer compared to healthy controls. However, they only defined MDSCs as Lin-HLA-DR-CD33+ with no further characterization of granulocytic or monocytic subsets.

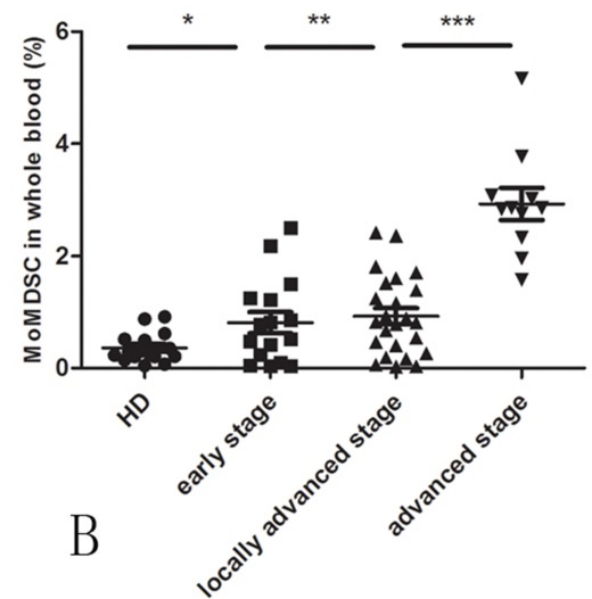

Fig 2. The level of MDSCs in cervical cancer patients. (A) The frequency of GrMDSCs increased with the increase of clinical cancer stage. (B)The frequency of MoMDSCs increased only in advanced cancer stage. 

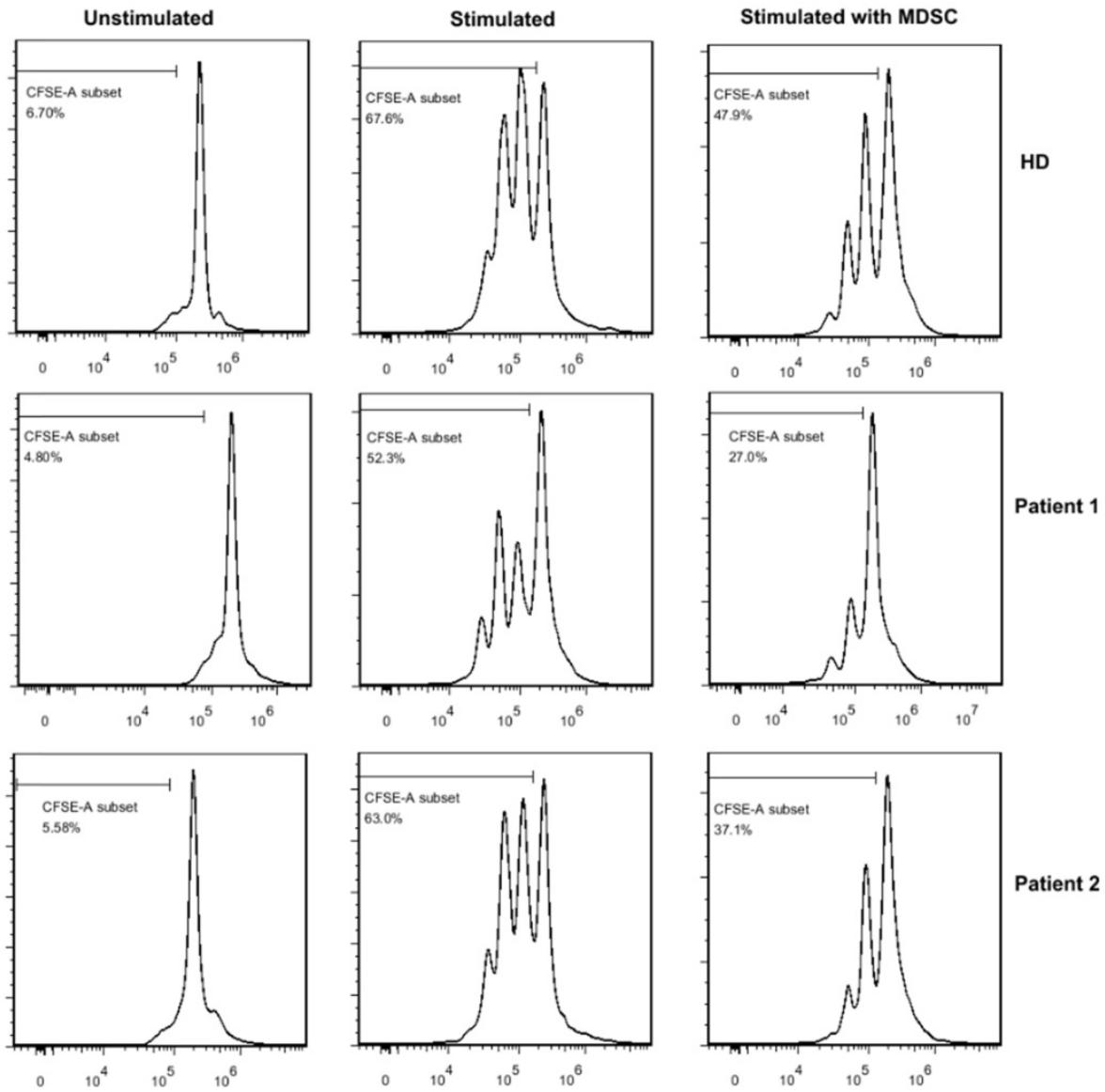

Fig 3. Suppression of T cell proliferation in vitro. When sorted MDSCs were cocultured with CFSE-labeled autologous $\mathrm{T}$ cells,the proliferation index of $\mathrm{T}$ cells reducing to $39.9 \%$ and $58.9 \%$ respectively in two cancer patients, while the $T$ cell proliferation index was $91.6 \%$ in healthy donor.

Our study demonstrated that both subsets were significantly increased. Similar with our research, the frequency of both MoMDSCs and GrMDSCs were significantly elevated in the blood of patients from advanced glioma [9]. In gastric cancers [10] or pancreatic cancers [11], only the level of GrMDSCs subtype was elevated while in prostate cancers, significant elevated MDSCs frequency was seen only in CD14+HLA-DR-/low MoMDSCs [12]. These findings as well as the MDSC plasticity in mice [13] indicated that release of tumor-derived factors in different tumors may cause the expansion of particular type of MDSCs.

We showed that significant increasing GrMDSCs levels were seen in patients with increasing tumor grade as well as known adverse prognostic factors such as metastatic lymph nodes, deep stromal invasion. Moreover, the higher frequency of GrMDSCs was seen in recurrent patients than in non-recurrent patients. These observations indicated that circulating GrMDSCs expansion might facilitate tumor growth and progression in cervical cancers. Previous studies on other cancers showed consistent findings. Stanojevic etc. [14] found that the GrMDSCs frequency correlated well with the clinical stage of cutaneous melanoma and the highest frequency emerged in stage IV melanoma patients. In prostate cancers [15] and colorectal cancers [16], patients with lymph node metastases or poor prognosis also had significantly more circulating MDSCs. We failed to reveal the associations between the clinical parameters or prognosis and MoMDSCs. This may be due to these samples are confined to early and locally advanced cervical cancer patients and MoMDSCs have not fully expanded yet and therefore, cannot reflect the tumor burden in this period.

An interesting finding in our experiment was that, the frequency of GrMDSCs elevated in cervical cancer of stage $\mathrm{Ib} 2$, indicating that accumulation of these cells was a relatively early event in tumor development. We speculate that the sustained HPV-E6/E7 expression during tumor progression may play an important role in the immunological pathogenesis including MDSC stimulation. Some studies also showed the elevated frequency of GrMDSCs in early stage, such as breast cancers [17] and cutaneous melanomas [14]. We noted that lysed peripheral blood sample was used in these studies, which may prevent possible loss of the GrMDSCs. 
MDSCs have the capacity to suppress the adaptive immune response mediated by $\mathrm{T}$ cells, but these findings were not well established in cervical cancer patients. To our knowledge, this is the first study to confirm that the relation between circulating MDSCs cells and CD8+ $T$ cells in local immuno-environment in cancer patients. The weak negative linear relationship between circulating GrMDSCs and infiltrating CD8+ cells implicated that the accumulation of cytotoxic lymphocytes in the tumor environment could be suppressed in the context of high circulating GrMDSCs, thus resulting in the decreased local anti-tumor immunological effect. MDSCs was found to modulate the development and induction of Treg cells recently [15], however our study failed to show the relationship between MDSCs and T cells, implicating the complex mechanisms underlying the proliferation of Treg cells, in which other than MDSCs might be involved.

Although various markers have been proposed to define MDSCs, the most definitive identification of MDSC still remains their immunosuppressive function. We found that the GrMDSCs were able to inhibit $T$ cell proliferation in vitro. In addition, the inhibition capacity was more powerful in cancer patients than those isolated from healthy donor. This result proved that GrMDSCs from cervical cancer patients have $\mathrm{T}$ cell immunosuppressive effects.

There are several factors can influence the quantification of circulating MDSCs. First, the choice either PBMCs or WB as the source for circulating MDSCs can influence results and may lead to potential bias in the evaluation of granulocytic cell subset [18]. Loss of high-density polymorphonuclear neutrophil (HD-PMN) in PBMCs during the process of the Ficoll density gradient separation can result in a partial loss of GrMDSCs [19]. In our study, we used whole blood to analyze the percentage circulating MDSCs, which could precisely reflect the original distribution of immune cell populations in the tested sample. Second, cell components were more complex in whole blood than in PBMCs. The large number of neutrophil cells in whole blood requires additional immuno-labels to exclude nonspecific staining. A lineage of cocktails (CD3/ CD19/ CD56/ CD14/ CD20/ CD16) was applied in our experiment. The negative selection of CD16 antibody could filter the mature neturophils out. Mature lymphocytes were negatively selected by CD3/CD19/CD56 antibodies and mononuclear cells by CD14. Third, there is no well-established combination of markers defining the subset of MDSCs although they are broadly separated into CD15+GrMDSCs and CD14+MoMDSCs subtypes. Combinations such as HLA-DR- CD33+ CD11b+ CD14- CD15+, CD11b+ CD14- HLA-DR-
CD33+ CD15+, CD33+ HLA-DR- CD15+, CD11b+ CD14- CD15+, CD11b+ CD14- CD33+, Lin- CD33+ $\mathrm{CD} 11 \mathrm{~b}+\mathrm{CD} 15+$ etc. were applied to define GrMDSCs [20], but its precision was not well documented. In this study, we applied a stringent definition with the applications of more markers for sorting subpopulations. We believe that these technical points are one of the major advantages in our study. Another limitation is the heterogeneity in adjuvant post-operative therapies, which might affect survival outcome in NACT patients with risk factors. However, several studies reported equivalent therapeutic results between adjuvant chemotherapy, chemoradiotherapy, and radiotherapy after radical surgery [21,22].

In conclusion, our study demonstrates that the increased frequencies of circulating GrMDSCs are associated with tumor burden and recurrence in early and locally advanced cervical cancer patients. These findings, together with the decreased CD8+ cells numbers in local immuno-environment, as well as the inhibited $\mathrm{CD} 8+\mathrm{T}$ cell proliferation index in vitro support the immunosuppressive functions of GrMDSCs in cervical cancer patients. Our study suggests the GrMDSCs can serve as a potential biomarker for evaluating the disease progression in cervical cancers.

\section{Abbreviations}

MDSCs: Meloid-derived suppressor cells; PBMCs: Peripheral blood mononuclear cells; CFSE: Carboxy fluorescein succinimidy ester; TIL: Tumor infiltrating lymphocyte; DFS: Disease-free survival; NACT: New adjuvant chemotherapy; pathological optimal response: pOR; polymorphonuclear neutrophil: PMN.

\section{Supplementary Material}

Supplementary figure.

http://www.jcancer.org/v10p1341s1.pdf

\section{Acknowledgements}

This study was funded by National Key R\&D Program of China (2016YFC1302900).

\section{Competing Interests}

The authors have declared that no competing interest exists.

\section{References}

1. Shipp C, Speigl L, Janssen N, et al. A clinical and biological perspective of human myeloid-derived suppressor cells in cancer. Cellular and molecular life sciences: CMLS. 2016;73(21):4043-61

2. Youn JI, Nagaraj S, Collazo M, et al. Subsets of myeloid-derived suppressor cells in tumor-bearing mice. Journal of immunology (Baltimore, Md : 1950). 2008;181(8):5791-802. 
3. Talmadge JE, Gabrilovich DI. History of myeloid-derived suppressor cells. Nature reviews Cancer. 2013;13(10):739-52.

4. Ostrand-Rosenberg S, Sinha P. Myeloid-derived suppressor cells: linking inflammation and cancer. Journal of immunology. 2009;182(8):4499-506.

5. Lechner MG, Megiel C, Russell SM, et al. Functional characterization of human $\mathrm{Cd} 33+$ and $\mathrm{Cd} 11 \mathrm{~b}+$ myeloid-derived suppressor cell subsets induced from peripheral blood mononuclear cells co-cultured with a diverse set of human tumor cell lines. Journal of translational medicine. 2011;9:90.

6. Kawano M, Mabuchi S, Matsumoto $Y$, et al. The significance of G-CSF expression and myeloid-derived suppressor cells in the chemoresistance of uterine cervical cancer. Scientific reports. 2015;5:18217.

7. Van Hede D, Langers I, Delvenne P, et al. Origin and immunoescape of uterine cervical cancer. Presse medical. 2014;43:e413-21.

8. Song $\mathrm{D}, \mathrm{Li} \mathrm{H}, \mathrm{Li} \mathrm{H}$,et al. Effect of human papillomavirus infection on the immune system and its role in the course of cervical cancer. Oncology letters. 2015;10(2):600-6.

9. Gielen PR, Schulte BM, Kers-Rebel ED, et al. Elevated levels of polymorphonuclear myeloid-derived suppressor cells in patients with glioblastoma highly express S100A8/9 and arginase and suppress $\mathrm{T}$ cell function. Neuro-oncology. 2016;18(9):1253-64.

10. Wang L, Chang EW, Wong SC, et al. Increased myeloid-derived suppressor cells in gastric cancer correlate with cancer stage and plasma S100A8/A9 proinflammatory proteins. Journal of immunology (Baltimore, Md : 1950). 2013;190(2):794-804

11. Khaled YS, Ammori BJ, Elkord E. Increased levels of granulocytic myeloid-derived suppressor cells in peripheral blood and tumour tissue of pancreatic cancer patients. Journal of immunology research. 2014;2014:879897.

12. Brusa D, Simone M, Gontero $\mathrm{P}$, et al. Circulating immunosuppressive cells of prostate cancer patients before and after radical prostatectomy: profile comparison. International journal of urology : official journal of the Japanese Urological Association. 2013;20(10):971-8.

13. Youn JI, Kumar V, Collazo M, et al. Epigenetic silencing of retinoblastoma gene regulates pathologic differentiation of myeloid cells in cancer. Nature immunology. 2013;14(3):211-20.

14. Stanojevic I, Miller K, Kandolf-Sekulovic L, et al. A subpopulation that may correspond to granulocytic myeloid-derived suppressor cells reflects the clinical stage and progression of cutaneous melanoma. International immunology. 2016;28(2):87-97.

15. Idorn M, Kollgaard T, Kongsted $\mathrm{P}$, et al. Correlation between frequencies of blood monocytic myeloid-derived suppressor cells, regulatory $\mathrm{T}$ cells and negative prognostic markers in patients with castration-resistant metastatic prostate cancer. Cancer immunology, immunotherapy : CII. 2014;63(11):1177-87.

16. Zhang $\mathrm{B}$, Wang $\mathrm{Z}, \mathrm{Wu} \mathrm{L}$, et al. Circulating and tumor-infiltrating myeloid-derived suppressor cells in patients with colorectal carcinoma. PloS one. 2013;8(2):e57114.

17. Diaz-Montero CM, Salem ML, Nishimura MI, et al. Increased circulating myeloid-derived suppressor cells correlate with clinical cancer stage, metastatic tumor burden, and doxorubicin-cyclophosphamide chemotherapy. Cancer immunology, immunotherapy : CII. 2009;58(1):49-59.

18. Damuzzo V, Pinton L, Desantis G, et al. Complexity and challenges in defining myeloid-derived suppressor cells. Cytometry Part B, Clinical cytometry. 2015;88(2):77-91.

19. Grutzner E, Stirner R, Arenz L, et al. Kinetics of human myeloid-derived suppressor cells after blood draw. Journal of translational medicine. 2016;14:2.

20. Umansky V, Blattner C, Gebhardt C, et al. The Role of Myeloid-Derived Suppressor Cells (MDSC) in Cancer Progression. Vaccines. 2016;4(4):1-16.

21. Wen HW, Huang WP, Liu TY, Ma K, Tao X, Zhu LR, et al. Results of different postoperative adjuvant therapies for stage Ib-IIa cervical carcinoma with risk factors. Zhonghua Fu Chan Ke Za Zhi. 2013 ;48(12):920-4.

22. Lee KB, Lee JM, Ki KD, Lee SK, Park CY, Ha SY. Comparison of adjuvant chemotherapy and radiation in patients with intermediate risk factors after radical surgery in FIGO stage IB-IIA cervical cancer. Int J Gynecol Cancer. 2008 ;18(5):1027-31 